\title{
Role of Matrix Metalloproteinases 2 in Spinal Cord Injury-Induced Neuropathic Pain
}

\author{
Gurwattan S. Miranpuri Dominic T. Schomberg Bahauddeen Alrfaei \\ Kevin C. King Bryan Rynearson Vishwas S. Wesley Nayab Khan \\ Kristen Obiakor Umadevi V. Wesley Daniel K. Resnick
}

Department of Neurological Surgery, University of Wisconsin School of Medicine and Public Health, Madison, Wis., USA

\section{Key Words}

Matrix metalloproteinases - $\beta$-Catenin · ERK/MAPK .

Contusion spinal cord injury $\cdot$ Neuropathic pain .

Thermal hyperalgesia

\begin{abstract}
Neuropathic pain (NP) affects approximately 4 million people in the United States with spinal cord injury (SCI) being a common cause. Matrix metalloproteinases (MMPs) play an integral role in mediating inflammatory responses, cellular signaling, cell migration, extracellular matrix degradation and tissue remodeling and repair. As such, they are major components in the pathogenesis of secondary injury within the central nervous system. Other gene regulatory pathways, specifically MAPK/extracellular signaling-regulated kinase (ERK) and Wnt/ $\beta$-catenin, are also believed to participate in secondary injury likely intersect. The study aims to examine the MMP-2 signaling pathway associated with ERK and $\mathrm{Wnt} / \beta$-catenin activity during contusion $\mathrm{SCl}(\mathrm{CSCl})$-induced NP in a rat model. This is an experimental study investigating the implication of MMP- 2 in SCl-induced NP and its association with the cellular and molecular changes in the interactions between extracellular signaling kinase and
\end{abstract}

$\beta$-catenin. Adult Sprague-Dawley rats received cSCl injury by NYU impactor by dropping $10 \mathrm{~g}$ weight from a height of 12.5 $\mathrm{mm}$. Locomotor functional recovery of injured rats was measured on post $\mathrm{CSCl}$ day 1 , and weekly thereafter for 6 weeks using Basso, Beattie and Bresnahan scores. Thermal hyperalgesia (TH) testing was performed on days $21,28,35$ and 42 post $\mathrm{CSCl}$. The expression and/or activity of MMP-2, $\beta$-catenin and ERK were studied following harvest of spinal cord tissues between 3 and 6 weeks post $\mathrm{cSCl}$. All experiments were funded by the department of Neurological Surgery at the University of Wisconsin, School of Medicine and Public Health having no conflict of interest. MMP- 2 and $\beta$-catenin expression were elevated and gradually increased from days 21 to 42 compared to sham-operated rats and injured rats that did not exhibit TH. The expression of phosphorylated ERK (phospho-ERK) increased on day 21 but returned to baseline levels on day 42 whereas total ERK levels remained relatively unchanged and constant. Chronic NP is associated with changes in the expression of MMP-2, $\beta$-catenin and ERK. Our data suggest that the transient upregulation of phospho-ERK is involved in the initial upregulation of both $\beta$-catenin and MMP-2 following CSCl-induced NP states.

(c) 2016 S. Karger AG, Basel

Daniel K. Resnick, MD, MS

Department of Neurological Surgery, University of Wisconsin School of Medicine and Public Health, Box 8600, Clinical Science Center, 600 Highland Ave Madison, WI 53792 (USA)

E-Mail resnick@ neurosurgery.wisc.edu
2016 S. Karger AG, Basel

0972-7531/16/0231-0025\$39.50/0 


\section{Introduction}

More than 1 million people suffer from spinal cord injury (SCI) in the United States alone with more than 12,000 new cases each year. The annual global incidence is $15-40$ cases per million [1]. Currently, no globally acceptable treatment exists for SCI-induced neuropathic pain (NP). NP is a common outcome following SCI occurring in up to $70 \%$ of SCI patients [2-5]. In the context of SCI, secondary injury is characterized by sustained inflammatory activity that results in exacerbation of locomotor deficits and abnormal nociception [6]. An imperative upstream modulator of inflammatory cytokine production is the matrix metalloproteinase (MMP) system that cleaves the pro-form of cytokines to the active form [7]. Peripheral nerve injury (PNI) and SCI share many of the same mediators of neuroinflammation and pain induction [8].

We chose to examine the role of MMP-9 and MMP-2 in our rat model of contusion SCI (cSCI) as it relates to the inception of NP. MMP-9 and MMP-2 have been reported to contribute to the breakdown of the extracellular matrix in the acute and chronic phases following SCI, respectively [9]. MMPs also function as inflammatory mediators by cleaving pro-form cytokines into their active form. In a PNI model, MMP-9 induces NP through interleukin-1 $\beta$ (IL-1 $\beta$ ) cleavage as well as by microglia activation. MMP-2 appears to play a role in the persistence of NP through on-going IL- $1 \beta$ cleavage and induced astrocyte activation and proliferation in the late phase of injury [7-10].

Extracellular signaling-regulated kinase (ERK) pathway activation within the dorsal horn of the spinal cord correlates with the development of thermal and mechanical hyperalgesia in rats following intra-plantar injection of BMK scorpion venom [11]. Upregulation of MMP-2 is initiated through specific signal transduction pathways and the ERK pathway is an example of one such pathway, which may be involved in upregulating MMP-2 after SCI.

Early upregulation of $\beta$-catenin in the spinal cord dorsal horn after PNI has also been reported [12]. $\beta$-Catenin activity has also been shown to directly result in MMP-2 and MMP-9 gene upregulation [13]. The MAPK/ERK and $\mathrm{Wnt} / \beta$-catenin pathways do not operate in isolation. Communication between these pathways has been documented within cancer studies, but has not been addressed in SCI-induced NP models [10]. Wnts are able to strongly induce activation of MAPK intermediates [14]. Our rationale for this study comes from the associations between MMP activity and signaling pathways known to be involved in NP. The goal of this study was to examine relationships between cSCI-induced NP states and timespecific changes inMMP-2, $\beta$-catenin and ERK following SCI.

\section{Methods}

Animals Used

Adult male Sprague-Dawley rats $(\mathrm{n}=30)$ weighing 275-300 g were used for these experiments. All experiments were conducted following the guide for the care and use of laboratory animals and were approved by the institutional animal care and utilization committee at the University of Wisconsin School of Medicine and Public Health, Madison, Wis., USA.

\section{Spinal Cord Injury}

Following acquisition of baseline data, laminectomy was performed on the rats to produce SCI at spinal level T9, following the induction of adequate inhalational anesthesia (Isoflurane, induction $5 \%$, maintenance $2.5 \%$, in a 50:50 mixture of oxygen and nitrous oxide). Adult male Sprague-Dawley rats (275-300 g) underwent cSCI, a method employed in our laboratory since 2003 for inducing cSCI by NYU Impactor Model II (W.M. Keck Center for collaborative Neuroscience Rutgers, State University of New Jersey), with $12.5 \mathrm{~g} / \mathrm{cm}$ [15-24]. The NYU impactor is used because it is the standard for reliable and reproducible impaction injuries in rat models. Additional sham-operated controls underwent laminectomies, but were not contused. The impaction produced transient paralysis in the hind limbs. Following surgery, all of the rats underwent manual bladder expression until bladder control was re-established. All rats also received $50 \mathrm{mg} / \mathrm{kg}$ of cefazolin by subcutaneous injection for bladder infection prophylaxis for 7 days following surgery. This protocol is in accordance with the NIH guidelines and has been approved by the University of Wisconsin Institutional Animal Care and Use Committee.

\section{Locomotor Functional Recovery}

The functional neurological deficits were assessed by behavioral analysis based on the Basso, Beattie and Bresnahan (BBB) open-field locomotors test [25], a method routinely used in our lab. The rats were placed into an open field testing area and were assigned numbers based on their functional recovery. The scores can range from 0 (no hind limb movement) to 21 (normal movement and coordinated gait with parallel paw placement). A BBB score of 15 or more showing 'consistent plantar stepping and consistent front limb-hind limb coordination, no toe clearance or occasional toe clearance during forward limb advancement, predominant paw position is parallel to the body at initial contactor' was deemed necessary for an animal to be tested for thermal hyperalgesia (TH). BBB scores were assessed prior to injury (baseline) and then again on days $21,28,35$ and 42 post-SCI/post-sham surgery. The animals were recorded by video for $4 \mathrm{~min}$ and then scored in a blinded fashion.

\section{Assessment of TH}

TH testing developed by Hargreaves et al. [26] assesses that an animal's withdrawal of a hind paw to a thermal noxious stimulus has been adopted in our lab since 2006 [15-24]. Also, in our 
lab, we have developed a valid and useful pain index in 979 Sprague-Dawley rats [23] used as an accurate index for hyperalgesia to thermal stimuli in rats subjected to T9 contusive SCI. Briefly, rats were placed in an apparatus (Plantar ${ }^{\mathrm{TM}}$ Test, Stoelting, Ill., USA) with a moveable focused beam of heat from an infrared emitter that is placed under one of the hind paws. The heat from the apparatus was slowly increased until the animal moved its hind paw. When the hind limb is withdrawn the photocell automatically shuts off and the latency time for the animal to lift its hind paw was recorded. A safety cutoff of $20 \mathrm{~s}$ is used so that the animal does not receive prolonged exposure to the heat. Most baseline tests result in a latency of 8-12 s. After SCI, a decrease in latency of $3 \mathrm{~s}$ or more was considered to signify $\mathrm{TH}$. These TH testing were repeated on days $21,28,35$ and 42 following surgery.

\section{Spinal Cord Harvesting}

The epicenter and rostral regions of the contused spinal cords were harvested. The sections were immediately submerged in liquid nitrogen and stored at $-80^{\circ} \mathrm{C}$. Spinal cord samples were harvested from $\mathrm{TH}+, \mathrm{TH}-$, and sham-operated rats on days $21,28,35$ and 42 following SCI.

\section{Zymography}

Activity of MMP-2 was measured based on its ability to hydrolyze gelatin protein substrate within the gel. The zymogram measures MMP-2 enzyme activity in each sample through MMP-2-specific staining following the electrophoresis. For gelatin zymography, $10 \mu \mathrm{g}$ protein from each tissue supernatant was applied to $10 \%$ SDS-PAGE gels copolymerized with type-A gelatin (1 mg/ml; Sigma Aldrich). After electrophoresis, gels were washed in $2.5 \%$ Triton X-100, incubated for $18 \mathrm{~h}$ at $37^{\circ} \mathrm{C}$ and stained in $0.1 \%$ Coomassie brilliant blue. The gelatinolytic regions were observed as white bands against a blue background.

\section{Western Blot Analysis for MMP-2, ERK and $\beta$-Catenin}

SDS-PAGE was employed for blot analysis. Preparation of samples for loading: the standard loading $2 \times$ Laemmli buffer: $4 \%$ SDS, $10 \%$ 2-mercaptoehtanol, $20 \%$ glycerol, $0.004 \%$ bromophenol blue, $0.125 \mathrm{M}$ Tris-HCl. Using special gel loading tips, samples were loaded into each well. Thirty micrograms of total protein was separated by SDS-PAGE. The gels were submerged in running buffer, which contains $25 \mathrm{mM}$ Tris base, $190 \mathrm{mM}$ glycine and $0.1 \%$ SDS. Gels were run for $1 \mathrm{~h}$ and power was turned off when the migration front reached the bottom of the gel. Then the membranes were blocked using $5 \%$ milk powder in $1 \times$ TBST (Tris buffer, sodium chloride, Tween 20). Blocking the membrane prevents non-specific background binding of the primary and/or secondary antibodies to the membrane. After blocking, membranes were incubated with respective primary antibodies (incubation buffer: $1 \times$ TBST with $5 \%$ milk and diluted primary antibody (total ERK or phosphorylated ERK (phospho-ERK) or $\beta$-catenin and control $\beta$-actin)) overnight in cold room with agitation to enable homogenous covering of the membrane and to prevent uneven binding. Membranes were washed several times in TBST while agitating, 5 min per wash, to remove residual primary antibodies, and membranes were then incubated in secondary antibody containing horse radish peroxidase enzyme for $1-2 \mathrm{~h}$, washed and developed us- ing enhanced chemiluminescent reagents and X-ray film. Automated X-ray film developers were used to visualize the protein bands.

\section{Statistical Methods}

Paired t test was used to compare the significance of differences between injured and non-injured groups of animals.

\section{Results}

We compared the expression of MMP-2, ERK and $\beta$-catenin in the spinal cord of animals exhibiting pain as determined by the presence of $\mathrm{TH}+$ following cSCI compared to injured animals with no pain ( $\mathrm{TH}-$ ) as well as sham-operated animals. Tissues were harvested on days 21 and 28 post cSCI depending upon the TH onset status. Higher MMP-2 expression, determined by Western blot analysis, was observed in $\mathrm{TH}(+)$ rats versus similarly injured $\mathrm{TH}(-)$. This elevation was seen beginning on day 21 and continued up to at least day 28 (fig. 1a). MMP-2 activity as indicated by zymography in $\mathrm{TH}(+)$ animals was shown to increase in subsequent days. MMP-9, on the other hand, declined over time (fig. 1b).

Phosphorylation of ERK1/2 following cSCI was increased only in $\mathrm{TH}+$ rats (fig. 1c). To evaluate changes in the ERK pathway, total and phospho-ERK1/2 were analyzed. Phospho-ERK upregulation was demonstrated via Western blot analysis with increased expression peaking on day 21. On day 28, phospho-ERK levels fell slightly and by day 42 , the levels had fallen to those similar to that of day 42 levels of $\mathrm{TH}(-)$ animals. Interestingly, only the phospho-ERK demonstrated this expression pattern while total-ERK levels remained constant on days 21,28 and 42 (fig. 1d).

Expression of $\beta$-catenin was upregulated following SCI. Western blot analysis confirmed the upregulated expression in $\mathrm{TH}(+)$ rats on day 21 . Levels continually increased peaking at day 42 (fig. 1e). However, $\beta$-catenin levels were elevated primarily in the injury epicenter and showed only weak elevation in the rostral region of spinal cord (fig. 1f).

\section{Discussion}

MMPs belong to a class of extracellular proteinases responsible for maintaining and remodeling the extracellular matrix. MMP expression and activity has been shown to play a role in the pathophysiology of traumatic brain injury [27]. The exact mechanism in which MMPs 


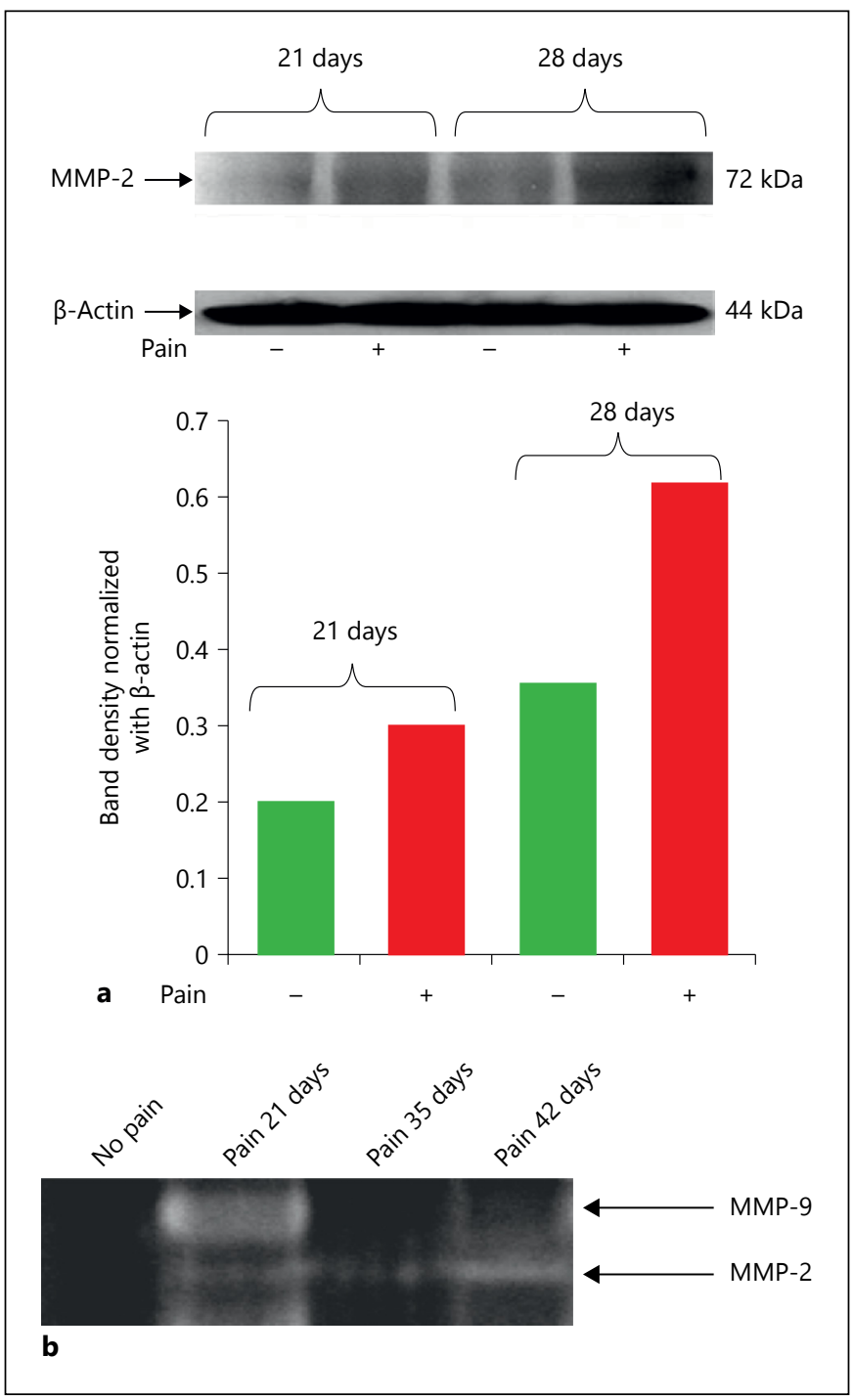

Fig. 1. Levels of MMP, ERK and $\beta$-catenin. a MMP-2 expression in pain (+) and no pain (-) animals on days 21 and 28 post cSCI. b Zymogram showing MMP-9 and MMP-2 activity on days 21, 35 and 42 following cSCI.

contribute to the SCI-induced pain is yet to be explained. However, the cannabinoid (CB) receptors and transient receptor potential vanilloid 1 (TRPV1) present a relationship between MMPs and nociceptive pathway. This is shown through agonism of $\mathrm{CB}$ receptors, which resulted in the reduction of $\mathrm{TH}$ after injury and induction of TIMPs, $[18,28-30]$ and CB2 receptors modulate the antihyperalgesic effect of WIN 55,212-2 in spinal cord injured $\mathrm{TH}+$ pain rats [22]. The activation of TRPV1 during cSCI-induced NP in rat models [17] and its implications in the expression of heat shock-induced MMP-1 expres- sion in human epidermal keratinocytes have been reported [30].

MMPs have been demonstrated to be involved in the activation of cell populations and the release of inflammatory cytokines following SCI [27, 31-36]. MMP-9 and MMP-2 contribute to the breakdown of the extracellular matrix following SCI and play a role in the induction and maintenance of peripheral nerve ligation models of $\mathrm{NP}$ [7]. MMP-9 induces NP through IL-1 $\beta$ cleavage as well as by microglia activation in the acute phase of injury, whereas MMP-2 is believed to sustain NP through IL- $1 \beta$ cleavage and induction of astrocyte activation and proliferation in the late stage of injury [7]. Inhibition of MMP-9 and MMP-2 in acute and late phase of injuries, respectively, employing TIMP1/2 and siRNA showed significant amelioration of NP in animals subject to spinal nerve ligation [7]. Our results are consistent with MMP-2 playing a similar role in SCI-mediated NP. Animal models have shown encouraging outcomes of several contributing factors including MMPs as contributors of tissue damage and repair but could not lead to successful clinical models as yet [37]. However, the emerging role of MMPs and TIMPs as pain modulators in alleviating NP, MMP-9 as pain inducer following SCI is of significance [38].

NP states are one of the most commonly reported symptoms in the chronic phase of SCI where animals with cSCI were tested for $\mathrm{TH}$ as the pain indicator. The selection of $3 \mathrm{~s}$ as a cutoff for the presence of TH was based on a statistically driven analysis of a database generated from a population of total 979 Sprague-Dawley spinal cord T9 injured rats used in our lab over the past 10 years [23]. This large data pool validates a statistically supported latency time in seconds to withdraw the hind paw, an indicator used as accurate index for hyperalgesia to thermal stimulus. ${ }^{1}$

Phospho-ERK (activated form of ERK) was upregulated after SCI injury in animals exhibiting TH pain. A significant difference was observed in phospho-ERK between animals in pain and those without pain. This suggests that phospho-ERK is involved in mediating the activity of MMP-2, which is a known contributor to the maintenance of NP. The decrease observed in phosphoERK expression on day 42 is consistent with a reduction in NP. Related research has shown that the metabotropic ATP receptor $\mathrm{P} 2 \mathrm{Y}(12)$ in microglia is involved in the activation of p38 MAPK, the production of cytokines and various pain mediators resulting in NP [39]. Microglia cells showed increased levels of $\mathrm{P} 2 \mathrm{Y}(12)$ protein and

\footnotetext{
${ }^{1}$ Global Neuroscience Initiative Foundation, Los Angeles, Calif., USA.
} 
Fig. 1. Levels of MMP, ERK and $\beta$-catenin. c Western blots showing total extra cellular signaling kinase ERK1/2 in epicenter (bottom row); phospho-ERK1/2 (top row) following cSCI. Ratios of phosphoERK1/2:total ERK1/2 shown graphically. d ERK $1 / 2$ is activated in animals with chronic pain on days 21 and 28 post cSCI. At day 42, the levels of ERK1/2 activation decreases to those similar to that of no pain animals. Graphical quantification of phospho-ERK1/2:total ERK1/2 also shown. e SCI increases $\beta$-catenin levels after 21 days with peak levels at day 42 as compared to sham. Quantitation of $\beta$-catenin also shown graphically.

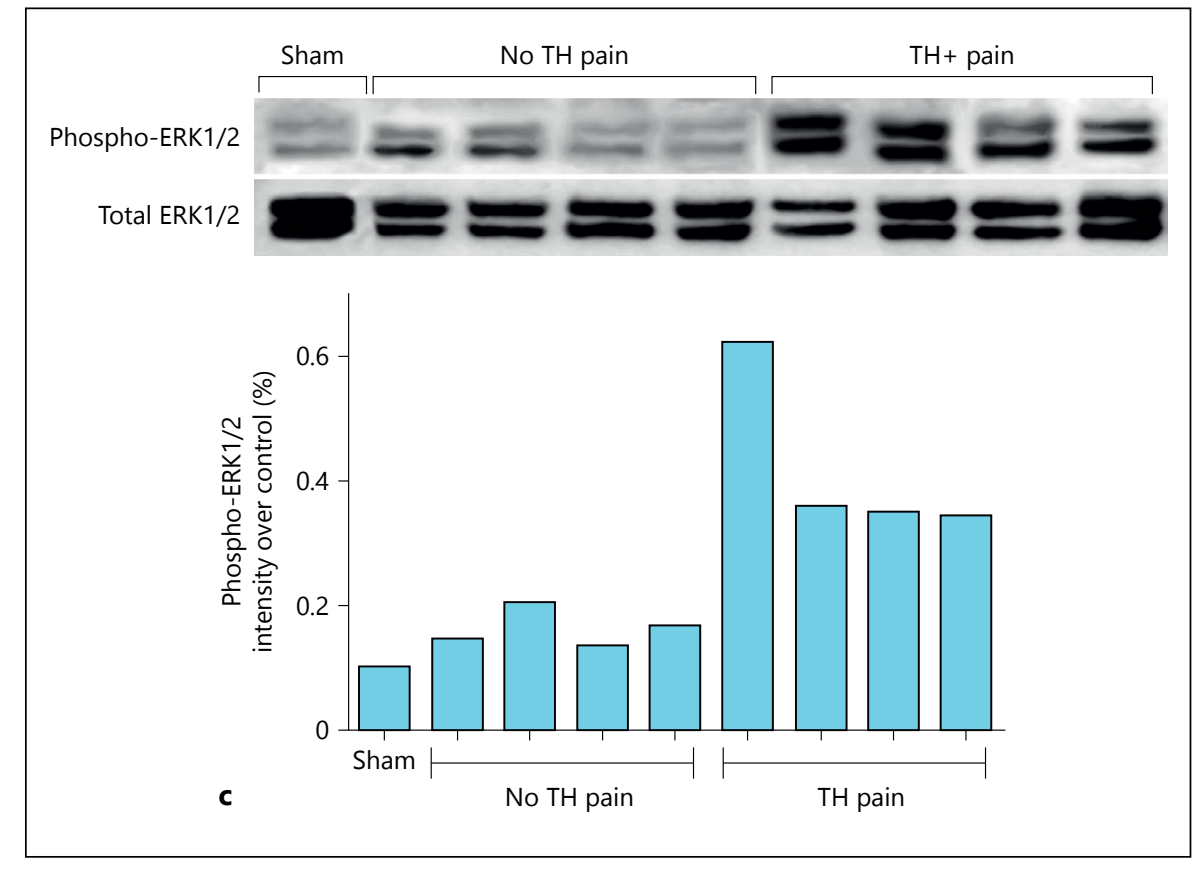

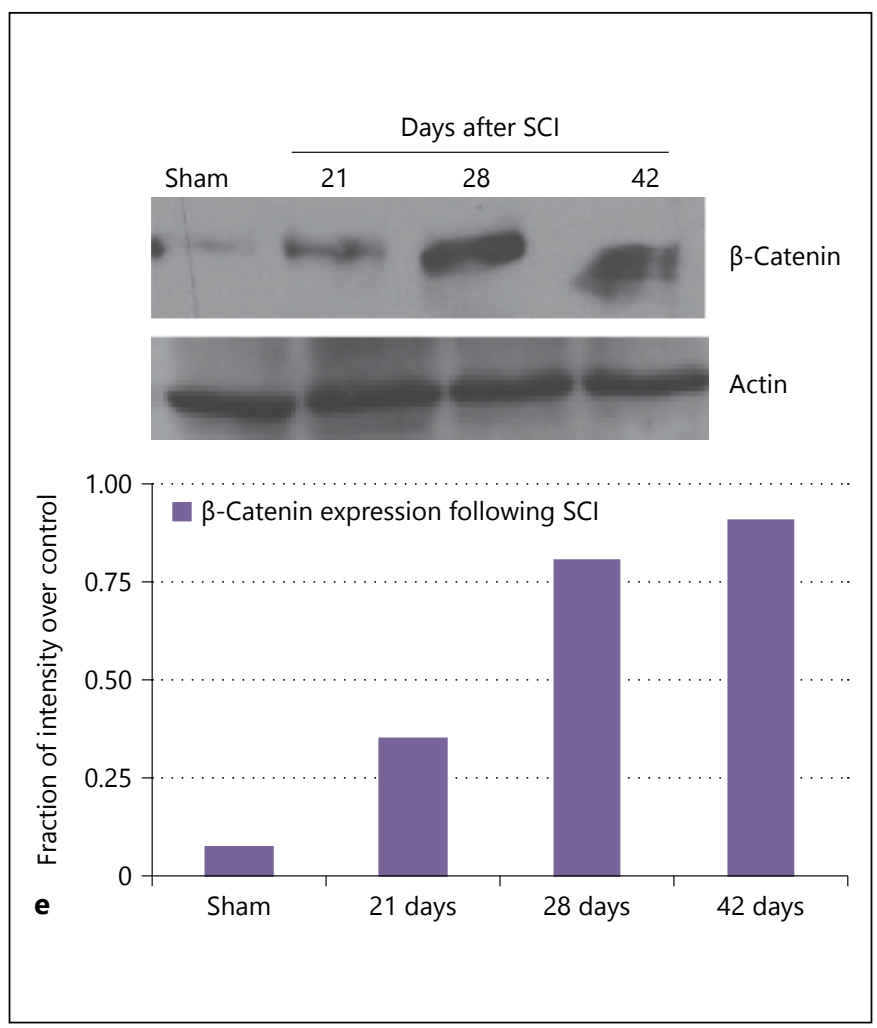

mRNA after SCI. By pharmacological antagonism and antisense knockdown of the P2Y(12) expression, both pain behavior and phosphorylation of p38 MAPK were reduced. This supports our findings that phosphorylation in the MAPK/ERK pathway plays a crucial role in the in-

duction of NP. Distinct role of MMP-9 and MMP-2 has been reported in a study employing spinal nerve ligation rat model [7]. During the acute phase of injury, ample expression of MMP-9 is required to induce NP via microglia activation and IL- $1 \beta$ signaling. On the other hand, 


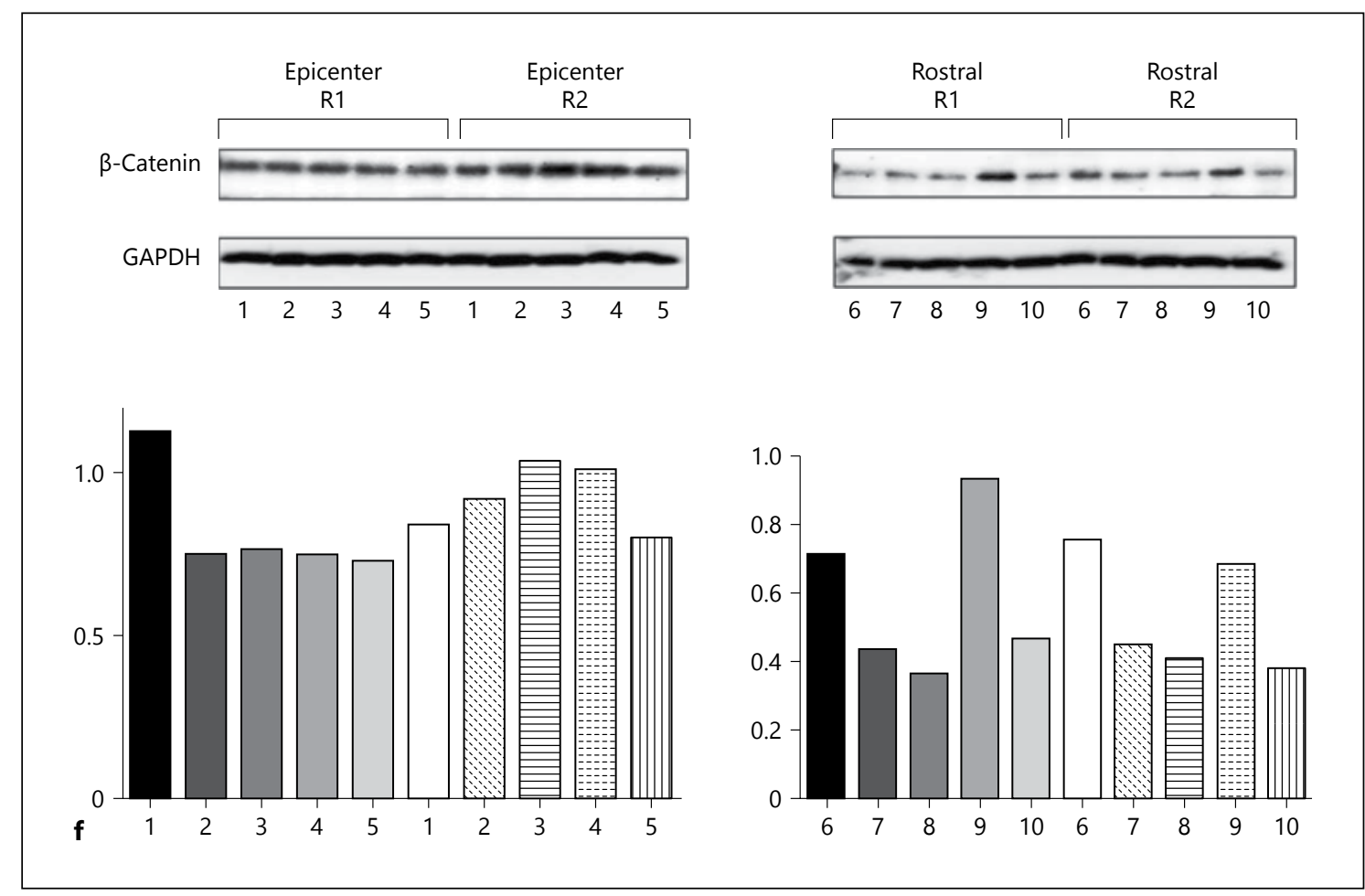

Fig. 1. Levels of MMP, ERK and $\beta$-catenin. $\mathbf{f} \beta$-Catenin expression in epicenter (1-5) and rostral region (6-10) of spinal cord following injury in $\mathrm{TH}(+)$ animals. 1-10 denote individual animal samples. Samples were run in duplicate $(\mathrm{R} 1=$ replicate $1, \mathrm{R} 2=$ replicate 2$)$.

during late phase of SNL, enhanced expression of MMP2 supports maintaining NP via IL- $1 \beta$ signaling and ERK activation in spinal astrocytes [7].

Further research has concluded that Wnt signaling plays a crucial role in the development of NP through the $\beta$-catenin-dependent pathway in the spinal cord [40]. They have reported that spinal blocking of the Wnt/Ryk receptor signaling inhibits persistent NP and blocking activation of this signaling system greatly reduces the hyper-excitability of sensory neurons and, thus, suppresses subsequent Ca-dependent signals, namely, CAMKII, Src, ERK, PKCy and CREB. These findings are in conjunction with our current research - targeting the Wnt/Ryk signaling system may be a viable strategy to alleviate NP.

We demonstrated a prolonged increase in $\beta$-catenin expression at days 21,28 and 42 following SCI compared to sham. In addition, $\beta$-catenin upregulation was detected in the epicenter region of injury but not in rostral region at day 42 after SCI compared to sham. While the time course of expression is more consistent with an important regulatory role of this pathway in MMP-2 expression, the rostral region always shows less expression compared to the epicenter injury area. It is possible that local activation of the $\beta$-catenin pathway influences a broader modification of MMP-2 activity. Further research is necessary to establish this link. It has been noted that injury of the astrocytes has been found to destabilize the cadherin-catenin complexes at the cellular membrane, which leads to nuclear translocation of $\beta$-catenin and diminished the response of astrocytes, which further corroborates our research [41]. Furthermore, the importance of the $\mathrm{Wnt} / \beta$-catenin signaling pathway is discussed, and its involvement in the regulation of the expression of a number of essential genes is critical for cell proliferation and differentiation [42]. The Wnt signaling pathway may also serve as a catalyst for disease progression and therapeutic targeting of this pathway may lead to alleviation of disease, which supports the conclusions of our study [43].

Ultimately, we intend to follow-up these studies with the use of specific inhibitors, including TIMPs, for the 3 aforementioned pathways. We anticipate that by determining the specific pathophysiological mechanisms for the development and maintenance of NP, we can design targeted delivery therapies for what currently remains an untreatable cause of suffering. 


\section{Acknowledgments}

Funding for this project was sponsored by the University of Wisconsin Hospital and Clinics Department of Neurosurgery. Special thanks to Dr. Robert Dempsey, Chairman, Department of Neurological Surgery, for constant encouragement throughout the duration of this research project.

\section{Disclosure Statement}

The authors declare that they have no competing interests.

\section{References}

1 National Spinal Cord Injury Statistical Center: The 2010 Annual Report for the Spinal Cord Injury Model Systems, National Spinal Cord Injury Statistical Center, Birmingham, Alabama, which is funded by grant number H133A060039 from the National Institute on Disability and Rehabilitation Research, Office of Special Education and Rehabilitative Services, U.S. Department of Education.

2 Finnerup NB, Sindrup SH, Jensen TS: Chronic neuropathic pain: mechanisms, drug targets and measurement. Fundam Clin Pharmacol 2007;21:129-136.

3 Yezierski RP: Spinal cord injury: a model of central neuropathic pain. Neurosignals 2005; 14:182-193.

4 Barrett H, McClelland JM, Rutkowski SB, Siddall PJ: Pain characteristics in patients admitted to hospital with complications after spinal cord injury. Arch Phys Med Rehabil 2003;84: 789-795.

5 Siddall PJ, McClelland JM, Rutkowski SB, Cousins MJ: A longitudinal study of the prevalence and characteristics of pain in the first 5 years following spinal cord injury. Pain 2003;103:249-257.

6 Noble LJ, Donovan F, Igarashi T, Goussev S, Werb Z: Matrix metalloproteinases limit functional recovery after spinal cord injury by modulation of early vascular events. J Neurosci 2002;22:7526-7535.

7 Kawasaki Y, Xu ZZ, Wang X, Park JY, Zhuang ZY, Tan PH, Gao YJ, Roy K, Corfas G, Lo EH, Ji RR: Distinct roles of matrix metalloproteases in the early- and late-phase development of neuropathic pain. Nat Med 2008;14:331336.

8 Detloff MR, Fisher LC, McGaughy V, Longbrake EE, Popovich PG, Basso DM: Remote activation of microglia and pro-inflammatory cytokines predict the onset and severity of below-level neuropathic pain after spinal cord injury in rats. Exp Neurol 2008;212:337-347.

9 de Castro RC Jr, Burns CL, McAdoo DJ, Romanic AM: Metalloproteinase increases in the injured rat spinal cord. Neuroreport 2000;11: 3551-3554.

10 Ji H, Wang J, Nika H, Hawke D, Keezer S, Ge Q, Fang B, Fang X, Fang D, Litchfield DW, Aldape K, Lu Z: EGF-induced ERK activation promotes CK2-mediated disassociation of alpha-catenin from beta-catenin and transacti- vation of beta-catenin. Mol Cell 2009;36:547559.

11 Pang XY, Liu T, Jiang F, Ji YH: Activation of spinal ERK signaling pathway contributes to pain-related responses induced by scorpion Buthus martensi Karch venom. Toxicon 2008;51:994-1007.

12 Zhang X, Chen G, Xue Q, Yu B: Early changes of beta-catenins and menins in spinal cord dorsal horn after peripheral nerve injury. Cell Mol Neurobiol 2010;30:885-890.

$13 \mathrm{Wu}$ B, Crampton SP, Hughes CC: Wnt signaling induces matrix metalloproteinase expression and regulates $\mathrm{T}$ cell transmigration. Immunity 2007;26:227-239.

14 Bikkavilli RK, Malbon CC: Mitogen-activated protein kinases and Wnt/beta-catenin signaling: molecular conversations among signaling pathways. Commun Integr Biol 2009;2:46-49.

15 Resnick DK, Cechvala CF, Yan Y, Witwer BP, Sun D, Zhang S: Adult olfactory ensheathing cell transplantation for acute spinal cord injury. J Neurotrauma 2003;20:279-285.

16 Resnick DK, Schmitt C, Miranpuri GS, Dhodda VK, Isaacson J, Vemuganti R: Molecular evidence of repair and plasticity following spinal cord injury. Neuroreport 2004;15:837-839.

17 Schmitt C, Miranpuri GS, Dhodda VK, Isaacson J, Vemuganti R, Resnick DK: Changes in spinal cord injury-induced gene expression in rat are strain-dependent. Spine J 2006;6:113119.

18 DomBourian MG, Turner NA, Gerovac TA, Vemuganti R, Miranpuri GS, Türeyen K, Satriotomo I, Miletic V, Resnick DK: B1 and TRPV-1 receptor genes and their relationship to hyperalgesia following spinal cord injury. Spine (Phila Pa 1976) 2006;31:2778-2782.

19 Rajpal S, Gerovac TA, Turner NA, Tilghman JI, Allcock BK, McChesney SL, Miranpuri GS, Park SW, Resnick DK: Antihyperalgesic effects of vanilloid-1 and bradykinin-1 receptor antagonists following spinal cord injury in rats. J Neurosurg Spine 2007;6:420-424.

20 Park SW, Yi JH, Miranpuri G, et al: Thiazolidinedione class of peroxisome proliferatoractivated receptor gamma agonists prevents neuronal damage, motor dysfunction, myelin loss, neuropathic pain, and inflammation after spinal cord injury in adult rats. J Pharmacol Exp Ther 2007;320:1002-1012.

21 Cramer SW, Baggott C, Cain J, Tilghman J, Allcock B, Miranpuri G, Rajpal S, Sun D,
Resnick D: The role of cation-dependent chloride transporters in neuropathic pain following spinal cord injury. Mol Pain 2008;4: 36.

22 Ahmed M, Rajpal S, Sweeney C, Gerovac TA, Allcock B, McChesney S, Patel AU, Tilghman JI, Miranpuri GS, Resnick DK: Cannabinoid subtype- 2 receptors modulate the antihyperalgesic effect of WIN 55,212-2 in rats with neuropathic spinal cord injury pain. Spine J 2010;10:1049-1054.

23 Kim HT, Kim T, Novotny B, Khan N, Aksamit J, Siegel S, Miranpuri GS, Resnick DK: Thermal hyperalgesia assessment for rats after spinal cord injury: developing a valid and useful pain index. Spine J 2014;14: 984-989.

24 Ahmed MM, Lee H, Clark Z, Miranpuri GS, Nacht C, Patel K, Liu L, Joslin J, Kintner D, Resnick DK: Pathogenesis of spinal cord injury induced edema and neuropathic pain: expression of multiple isoforms of WNK1. Ann Neurosci 2014;21:97-103.

25 Basso DM, Beattie MS, Bresnahan JC: A sensitive and reliable locomotor rating scale for open field testing in rats. J Neurotrauma 1995; 12:1-21.

26 Hargreaves K, Dubner R, Brown F, Flores C, Joris J: A new and sensitive method for measuring thermal nociception in cutaneous hyperalgesia. Pain 1988;32:77-88.

27 Wang X, Jung J, Asahi M, Chwang W, Russo L, Moskowitz MA, Dixon CE, Fini ME, Lo EH: Effects of matrix metalloproteinase- 9 gene knock-out on morphological and motor outcomes after traumatic brain injury. J Neurosci 2000;20:7037-7042.

28 Rice AS, Farquhar-Smith WP, Nagy I: Endocannabinoids and pain: spinal and peripheral analgesia in inflammation and neuropathy. Prostaglandins Leukot Essent Fatty Acids 2002;66:243-256.

29 Ramer R, Hinz B: Cyclooxygenase- 2 and tissue inhibitor of matrix metalloproteinases-1 confer the antimigratory effect of cannabinoids on human trabecular meshwork cells. Biochem Pharmacol 2010;80:846-857.

30 Li WH, Lee YM, Kim JY, Kang S, Kim S, Kim $\mathrm{KH}$, Park CH, Chung JH: Transient receptor potential vanilloid-1 mediates heat-shock-induced matrix metalloproteinase- 1 expression in human epidermal keratinocytes. J Invest Dermatol 2007;127:2328-2335. 
31 Parks WC, Wilson CL, López-Boado YS: Matrix metalloproteinases as modulators of inflammation and innate immunity. Nat Rev Immunol 2004;4:617-629.

32 Chattopadhyay S, Myers RR, Janes J, Shubayev V: Cytokine regulation of MMP-9 in peripheral glia: implications for pathological processes and pain in injured nerve. Brain Behav Immun 2007;21:561-568.

33 Rosenberg GA, Cunningham LA, Wallace J, Alexander S, Estrada EY, Grossetete M, Razhagi A, Miller K, Gearing A: Immunohistochemistry of matrix metalloproteinases in reperfusion injury to rat brain: activation of MMP-9 linked to stromelysin-1 and microglia in cell cultures. Brain Res 2001;893:104112.

34 Rosenberg GA: Matrix metalloproteinases in neuroinflammation. Glia 2002;39:279-291.
35 Yong VW: Metalloproteinases: mediators of pathology and regeneration in the CNS. Nat Rev Neurosci 2005;6:931-944.

36 Zhao BQ, Wang S, Kim HY, Storrie H, Rosen BR, Mooney DJ, Wang X, Lo EH: Role of matrix metalloproteinases in delayed cortical responses after stroke. Nat Med 2006;12:441445.

37 Ahmed MM, King KC, Pearce SM, Ramsey MA, Miranpuri GS, Resnick DK: Novel targets for spinal cord injury related neuropathic pain. Ann Neurosci 2011;18:162-167.

38 Lakhan SE, Avramut M: Matrix metalloproteinases in neuropathic pain and migraine: friends, enemies, and therapeutic targets. Pain Res Treat 2012;2012:952906.

39 Kobayashi K, Yamanaka H, Fukuoka T, Dai Y, Obata K, Noguchi K: P2Y12 receptor upregulation in activated microglia is a gateway of p38 signaling and neuropathic pain. J Neurosci 2008;28:2892-2902.
40 Liu S, Liu YP, Huang ZJ, Zhang YK, Song AA, Ma PC, Song XJ: Wnt/Ryk signaling contributes to neuropathic pain by regulating sensory neuron excitability and spinal synaptic plasticity in rats. Pain 2015;156:2572-2584.

41 Yang C, Iyer RR, Yu AC, Yong RL, Park DM, Weil RJ, Ikejiri B, Brady RO, Lonser RR, Zhuang $\mathrm{Z}$ : $\beta$-Catenin signaling initiates the activation of astrocytes and its dysregulation contributes to the pathogenesis of astrocytomas. Proc Natl Acad Sci U S A 2012;109: 6963-6968.

42 Takahashi-Yanaga F, Sasaguri T: The Wnt/ beta-catenin signaling pathway as a target in drug discovery. J Pharmacol Sci 2007;104: 293-302.

43 Maiese K, Li F, Chong ZZ, Shang YC: The Wnt signaling pathway: aging gracefully as a protectionist? Pharmacol Ther 2008;118:5881. 